\title{
Inadvertent plagiarism in young and older adults: The role of working memory capacity in reducing memory errors
}

\author{
DaVID P. MCCABE \\ Colorado State University, Fort Collins, Colorado \\ ANDERSON D. SMITH \\ Georgia Institute of Technology, Atlanta, Georgia \\ AND \\ Colleen M. Parks \\ University of California, Davis, California
}

\begin{abstract}
Two experiments examined inadvertent plagiarism in young and older adults. Young and older adults took turns generating category exemplars in small groups, and after a short retention interval recall was tested and subjects were asked to generate new exemplars (i.e., exemplars not initially generated). When asked to generate new exemplars, older adults were more likely to repeat exemplars that had been generated earlier by others (i.e., generatenew plagiarism). When asked to recall the exemplars they had generated earlier, older adults were more likely to claim that they had generated exemplars that had been generated by others (i.e., recall-own plagiarism), and were also more likely to falsely recall exemplars that had not been generated at all. There were no age differences in confidence for items that were plagiarized on the generate-new task. Hierarchical regression analyses indicated that age differences in generate-new plagiarism and false recall were entirely mediated by measures of episodic recall and working memory capacity. We conclude that inadvertent plagiarism errors result from the failure of systematic decision processes, and that controlled attention is important for avoiding memory errors.
\end{abstract}

In the introduction to their 1989 article on inadvertent plagiarism, Brown and Murphy quote B. F. Skinner (1983): "... [O]ne of the most disheartening experiences of old age is discovering that a point you have just made-so significant, so beautifully expressed — was made by you in something you published a long time ago" (p. 242). Skinner not only emphatically makes the point that plagiarism is a distressing event, but also suggests that it is more likely to occur in old age. Although there is now a substantial literature examining inadvertent plagiarism in young adults, this paper is the first to report experiments examining adult age differences in inadvertent plagiarism. Because aging is associated with selective declines in episodic memory processes, studying age differences in inadvertent plagiarism has the potential to explicate the factors influencing this sort of memory error.

\section{The Inadvertent-Plagiarism Paradigm}

Plagiarism is typically thought of as the intentional, fraudulent theft of another person's thoughts, words, or ideas (Mallon, 1989). However, as the quotation from Skinner suggests, plagiarism does not have to be intentional, and can occur without conscious awareness. This type of plagiarism, called inadvertent plagiarism, unconscious plagiarism, or cryptomnesia, occurs when people believe that thoughts, words, or ideas encountered previously are their own original creations (Brown \& Murphy, 1989). Inadvertent plagiarism can be fairly innocuous or it can have serious consequences. For example, you may be a bit embarrassed, after telling a joke, to realize that the person you told it to is the one who originally told it to you; or you may be subject to legal penalties after inadvertently copying a thought or idea recently published by a colleague.

Brown and Murphy (1989) have developed a procedure, used extensively to study this topic, for studying inadvertent plagiarism experimentally. There are typically three phases involved in this paradigm, and we will use the method employed in the original study to illustrate this procedure. In the initial generation phase, a small group of subjects was given a category cue (e.g., a type of fruit) and was asked to take turns generating exemplars (e.g., apple, peach, kumquat, etc.) from the category. Following a short delay, subjects were asked to recall the words that they themselves generated earlier (the recall-own phase). In a final phase, subjects generated a small number of new exemplars from each of the categories (the generate-new

D. P. McCabe, david.mccabe@colostate.edu 
phase). Subjects were instructed to provide only exemplars that had not already been generated. Inadvertent plagiarism was measured in each phase of this paradigm, operationalized in the initial-generation and generate-new phases as the proportion of responses that were repetitions of exemplars that were already generated (i.e., generatenew plagiarism). In the recall-own phase, inadvertent plagiarism was measured as the proportion of others' exemplars that were accidentally recalled as one's own (i.e., recall-own plagiarism). Reliable levels of inadvertent plagiarism were found in all three phases of the category generation task. The inadvertent-plagiarism paradigm has also been used to examine plagiarism using phonological categories (Brown \& Murphy, 1989), solutions in a word puzzle task (Marsh \& Bower, 1993), proper names (Tenpenny, Keriazakos, Lew, \& Phelan, 1998), and the generation of ideas (Marsh, Landau, \& Hicks, 1997).

Inadvertent plagiarism has typically been explained in the context of the source monitoring framework (Johnson, Hashtroudi, \& Lindsay, 1993), which suggests that memory accuracy is a joint function of the quality of the encoded information, the type and amount of information retrieved, and the decision processes used to evaluate the information retrieved. Memory errors often result from failure to discriminate between different sources of activation, a particularly likely outcome when these sources of activation are similar to one another. Support for the source monitoring framework explanation of inadvertent plagiarism comes from research showing that plagiarism in the different phases of the paradigm can be dissociated experimentally. Specifically, recall-own plagiarism is affected by variables that have deleterious effects on source memory, such as the similarity of sources, or distraction during initial generation, despite these variables having little effect on generate-new plagiarism (Landau \& Marsh, 1997; Macrae, Bodenhausen, \& Calvini, 1999). According to the source monitoring framework, these dissociations occur because the type of information that must be retrieved differs for the recall-own and generate-new task. Specifically, during the recall-own task, subjects must distinguish between self-generated items and items generated by others; this requires discrimination of different sources within the experimental context. By contrast, the generate-new task only requires subjects to differentiate between items that are old or new (i.e., item recognition; Brédart, Lampinen, \& Defeldre, 2003). Indeed, previous explanations of generate-new plagiarism suggest that these errors can be avoided by a simple assessment of familiarity; that is, if an exemplar is very familiar, it can be rejected as being old (Landau \& Marsh, 1997; Macrae et al., 1999). In summary, the recall task requires information that is qualitatively different from the information required on the generate-new task (Brédart et al., 2003; Landau \& Marsh, 1997; Macrae et al., 1999).

\section{Aging and Inadvertent Plagiarism}

Advancing adult age leads to declines in memory tasks that require conscious recollection of details of past events, such as those requiring source monitoring (see Balota, Dolan, \& Duchek, 2000, for a review). However, making familiarity-based recognition decisions, such as old-new recognition, often shows no difference as a function of age (Benjamin, 2001; Jennings \& Jacoby, 1993). Indeed, researchers often find this pattern within an experiment: age-related declines in source memory despite no age differences in old-new recognition (Brown, Jones, \& Davis, 1995; Naveh-Benjamin \& Craik, 1995). Based on these previous findings from the literature on aging, and on findings from the literature on inadvertent plagiarism, one might predict that aging would differentially affect recall-own plagiarism and thereby generate new plagiarism. On the basis of the voluminous literature demonstrating age-related declines in source monitoring (Glisky, Rubin, \& Davidson, 2001; Henkel, Johnson, \& De Leonardis, 1998; Johnson et al., 1993), most researchers would predict that older adults would be more likely than young adults to make recall-own plagiarism errors, as these errors reflect errors in monitoring source. However, on the basis of findings that recognition memory often does not differ as a function of age, coupled with the notion that the generate-new task is analogous to old-new recognition, one might predict no age difference in the likelihood of engaging in generate-new plagiarism. That is, if the cognitive processes involved in generate-new plagiarism only required a simple assessment of familiarity, it is quite possible that older adults would show levels of generate-new plagiarism similar to those of young adults. Support for this hypothesis comes from the aforementioned finding that distraction during initial generation increased recall-own plagiarism but left generate-new plagiarism unchanged (Macrae et al., 1999). Because aging typically leads to memory declines that are identical to disrupting the rehearsal of young adults (Castel \& Craik, 2003; Jennings \& Jacoby, 1993), the prediction that aging may selectively affect recall-own plagiarism, while having little effect on generate-new plagiarism, is tenable.

An alternative prediction with regard to age differences in inadvertent plagiarism is that older adults will show increased errors on both the recall-own and generate-new tasks. The generate-new task requires self-initiated retrieval, which, it could be argued, makes this task more similar to recall than to recognition (Craik \& Jennings, 1992). If this is the case, one would likely predict agerelated increases on the generate-new task. Furthermore, although previous research has established that the recallown and generate-new tasks differ in the type of information that must be retrieved, the two tasks may nonetheless require similar decision processes (Marsh et al., 1997).

According to the source monitoring framework, decision processes can be made heuristically or systematically (Johnson et al., 1993). Heuristic decisions are typically made quickly and are fairly automatic, whereas systematic decision processes are more time-consuming, deliberate, and effortful. Support for the notion that systematic decision processes are involved in the generate-new task comes from Marsh et al. (1997), who found that requiring subjects to respond quickly on the generate-new task resulted in increases in inadvertent plagiarism. They concluded that speeded responding reduced the likelihood that subjects could engage in systematic decision processes. Previous 
research indicates that aging and speeded responding often lead to similar decrements in performance (Jacoby, 1999; Light, Patterson, Chung, \& Healy, 2004), supporting the notion that aging may disrupt both generate-new task performance and recall-own plagiarism.

Other research on inadvertent plagiarism is consistent with the notion that aging may increase errors on both recall-own and generate-new tasks as well. For example, shallow levels of processing (i.e., determining word length) increased both recall-own plagiarism and generate-new plagiarism, compared with deeper levels of processing in young adults (i.e., a semantic orienting task; Marsh \& Bower, 1993). To the extent that aging mimics the effects of shallower levels of processing (Craik \& Jennings, 1992), one might expect increased inadvertent plagiarism of both types. A similar argument can be made for study-test delay. Delaying the recall-own and generatenew tasks increases the level of inadvertent plagiarism for both tasks (Brown \& Halliday, 1991; Marsh \& Bower, 1993). Previous research has established that young adults tested after a long study-test delay often show memory performance similar to older adults (Henkel et al., 1998; Schacter, Kaszniak, Kihlstrom, \& Valdiserri, 1991), again supporting the prediction of increased memory errors of both types for older adults.

The inadvertent-plagiarism findings reviewed thus far-indicating that shallower levels of processing, test delay, and disruption of effortful decision processes all increase generate-new plagiarism-are also consistent with a dual-process explanation of inadvertent plagiarism (Jacoby, 1999; Yonelinas, 2002). According to the dualprocess framework, memorial decisions are a joint function of two independent processes typically referred to as recollection and familiarity. According to the dual-process approach, memory errors often result because studying items increases their familiarity, but later these items cannot be recollected, leaving the effects of familiarity unopposed (Benjamin, 2001; Jacoby, 1999). Indeed, previous research using the lexical-decision task indicates that items that were plagiarized on the generate-new task were identified more quickly, and thus had higher levels of activation than those that were not (Marsh \& Landau, 1995). According to the dual-process framework, this increase in activation (i.e., enhanced familiarity) would be expected to lead to age-related increases in memory errors, at least to the extent that age-related declines in recollection leave this familiarity unopposed (Benjamin, 2001; Jacoby, 1999).

In addition to examining age differences in generatenew plagiarism, the category-generation paradigm we used in the present study (see Brown \& Murphy, 1989) offers an opportunity to examine age differences in false recall. Studying category exemplars often leads to false recall, presumably because studying many category exemplars enhances the familiarity of nonpresented exemplars from that category and makes discriminating presented and nonpresented items difficult (S. M. Smith, Gerkens, Pierce, \& Choi, 2002). Furthermore, a recent study by Meade and Roediger (2006) showed age-related increases in false recall in a similar category-recall paradigm. False recall in the inadvertent-plagiarism paradigm is particu- larly interesting, because it offers an opportunity to simultaneously examine whether aging affects memory errors in which new items are called old - that is, false recalland errors in which old items are called new-that is, generate-new plagiarism. We will also examine whether the nature of these memory errors is similar by examining individual differences in these errors.

\section{Mediators of Age-Related Increases in Memory Errors}

In order to better understand the nature of possible age differences in inadvertent plagiarism, we used an approach in which we supplemented the experimental data with analyses of individual differences (Butler, McDaniel, Dornburg, Price, \& Roediger, 2004; McCabe \& Smith, 2002; Sommers \& Huff, 2003). These hybrid designs, in which experimental and individual difference techniques are combined to examine the nature of remembering, are crucial to theory development in memory (Melton, 1967; Underwood, 1975), and crucial to understanding why age affects memory performance (A. D. Smith \& Earles, 1996). Simply knowing that aging increases memory errors is informative but does not reveal why aging is associated with increases in these errors. Others have noted that age-related increases in memory errors could occur because of general declines in episodic memory (Lövdén, 2003; Schacter, Israel, \& Racine, 1999), or these errors could result from age-related declines in more general cognitive resources, such as frontal-lobe functioning (Butler et al., 2004), inhibition (Lövdén, 2003), working memory capacity (McCabe \& Smith, 2002), or executive functioning (Rhodes \& Kelley, 2005).

The notion that episodic memory would be related to monitoring on the generate-new task is fairly straightforward: If you can remember which exemplars were presented during the initial-generation phase, you can reject these items as old on the generate-new task. A similar process would govern the rejection of new items on the recall task (i.e., false recall). That is, retrieval of studied items would allow new items to be rejected as such. Support for this idea comes from data showing that veridical recall is negatively correlated with false recall (e.g., Brainerd, Reyna, \& Kneer, 1995; Roediger, Watson, McDermott, \& Gallo, 2001).

The notion that retrieving studied exemplars can allow one to avoid generate-new plagiarism is intuitive, but there are other possible processes involved. For example, Balota et al. (1999) suggested that memory errors increase with age because of age-related declines in attentional control. These declines in attentional control lead to difficulty discriminating the activation arising from relevant versus irrelevant processing pathways. According to this point of view, when an attempt is made to generate new items, declines in attentional control make it difficult to discriminate between activation arising from the retrieval process itself and increased activation arising from having encountered an item during the study episode. Thus, memory errors are more likely for subjects such as older adults, who have declines in attentional control, because they cannot discriminate the source of currently activated information. Balota et al.'s (1999) hypothesis is very simi- 
lar to that of McCabe and Smith (2002), who suggested that working memory capacity, which they conceptualized as the ability to use controlled attention (see Engle, Tuholski, Laughlin, \& Conway, 1999), is required to discriminate between similar sources of activation. Although in a sample of older adults Balota et al. (1999) found nonsignificant correlations between purported measures of attentional control and false recall in the DRM paradigm (Deese, 1959; Roediger \& McDermott, 1995), McCabe and Smith found that greater working memory capacity was associated with reduced levels of false recognition in young and older adults (McCabe \& Smith, 2002), a finding consistent with a recent examination of false recall in young adults (Watson, Bunting, Poole, \& Conway, 2005). Thus, one would expect that better performance on a working memory capacity measure would be associated with less false recall, and to the extent that the monitoring processes required to avoid generate-new plagiarism are similar to those involved in avoiding false recall, the same relation may obtain between working memory capacity and generate-new plagiarism. In both cases, information encountered in the retrieval environment must be differentiated from information reactivated from the study context. Thus, in Experiments 1 and 2, subjects were administered a working memory capacity task, called Stroop span (McCabe, Robertson, \& Smith, 2005; McCabe \& Smith, 2002), and we examined whether performance on this measure, along with recall of category exemplars, was related to memory errors.

\section{EXPERIMENT 1}

The primary purpose of Experiment 1 was to examine whether older adults would show more inadvertent plagiarism on the generate-new task. To that end, we changed the typical ordering of the tasks in the paradigm, so that the generate-new task preceded the recall task. The order of tasks was initial generation, generate new, and recall. Using this test ordering eliminated the possibility that the recall phase would influence responding in the generatenew task, which was our primary measure of interest. Another change that we made was that subjects were asked after the generate-new phase to recall all of the exemplars that were generated during the initial-generation phase. We did this because each subject had only generated two exemplars during initial generation, and we wanted to avoid ceiling effects in recall.

\section{Method}

Subjects. Thirty-five undergraduates (mean age $=20.2, S D=$ 1.1 ) at the Georgia Institute of Technology participated for course credit. Thirty-three older adults (mean age $=73.7, S D=5.2$ ) were recruited from an ad in a local newspaper and were paid a small honorarium for their participation.

Materials and Procedure. Subjects in each age group were tested in groups of six. Each subject sat at a desk that had the letter A, B, C, D, E, or F printed on it. These desks were set up in two rows, facing the experimenter's desk. For three of the six groups of older adults, and for one of the six groups of young adults, a confederate (a graduate student) sat in as the sixth person, due to the absence of one subject. The confederates chose their responses from a printed sheet of exemplars, and they never plagiarized a response.
There were three basic phases of the experimental procedure: initial-generation, generate-new, and recall. In the initial-generation phase, subjects were informed that they would be given a category name (e.g., a type of fruit), and that they should generate an item from the category when the experimenter called their letter (e.g., Subject C). The experimenter read through an example category and the subjects completed an example category (an article of clothing) before beginning the initial-generation phase. Subjects were told that the order in which they would be called on would be randomly determined and that they would generate two items for each category. Subjects were also told not to repeat an item that had already been generated, but that if someone else happened to do this accidentally, no one should mention it. Each subject was called upon twice per category for a total of 12 items per category. Each category was completed before the next was started. The categories used, selected from the Battig and Montague (1969) norms, were sports, vegetables, musical instruments, metals, nonalcoholic beverages, and kitchen utensils. The experimenter wrote down each exemplar that had been generated. Subjects were told that the purpose of the study was to provide stimuli for another experiment. Thus, learning of the stimuli was incidental. Subjects were called on to generate exemplars in a prespecified order, following a Latin-square design. Subjects did not know the order in which they would be called on. All categories were included in each serial position once for each age group.

After completing $45 \mathrm{~min}$ of filler tasks (described below), subjects were asked to generate four new exemplars for each category (generate-new task). Subjects were given $12 \mathrm{~min}$ for this task, though no group used the whole time. After the generate-new task, subjects were asked to recall all 12 of the exemplars from each of the six categories from the initial-generation phase (recall task). Subjects were given $8 \mathrm{~min}$ for recall.

The subjects completed several other tasks during the interval between the initial-generation phase and the generate-new task. The tasks in order were Shipley vocabulary (Zachary, 1986), letter comparison (Salthouse \& Babcock, 1991), Stroop span (McCabe et al., 2005), and a task for which the subjects generated ideas (e.g., "How can school violence be reduced?"). A demographic questionnaire was given after the recall task. There was no apparent overlap between the stimuli on the interpolated tasks (vocabulary being the primary test that might have had some overlap) and the category exemplars used in the present experiments. These filler tasks, with the exception of the Shipley vocabulary task and Stroop span, were not analyzed as part of the present study.

For the Stroop span task, subjects were told that sequences of color words of varying length would be presented on the computer screen and that they should try to remember the color of each word presented until the word RECALL appeared, at which point they were to write down the first letter of each of the colors they had been remembering, in order, on the answer sheet provided. The word and font in which half of the color words were presented were congruent (e.g., the word RED in red font), and for the other half they were incongruent (e.g., the word BLUE in yellow font). Congruent and incongruent color words were mixed on each trial (see McCabe et al., 2005 , for a more detailed description). This task taxes working memory by requiring inhibition of the prepotent word reading response, while concurrently maintaining identified colors for recall. Previous research has established that performance on this task correlates with a more traditional measure of working memory capacity, as well as executive function measures (McCabe et al., 2005).

\section{Results and Discussion}

We used stringent scoring criteria to ensure that calculations of the proportion of errors for the generate-new task were based on the same denominator for all subjects. Because 10 young adults (29\%) and 5 older adults (16\%) did not generate four new words for the category "metal," this category was eliminated from the analyses. Of the five 
categories remaining, data from 3 young adults and 4 older adults were excluded because they did not generate four new responses for each category. A $p$ value of .05 was used for all analyses. (Note that having a confederate participate in some of the groups had no significant effect on responding for young or older adults on any of the memory measures; all pairwise comparisons had $p$ values $>.31$.)

Initial-generation phase. During the initial-generation phase of the experiment, when subjects were taking turns generating exemplars, they may have repeated exemplars that they had just heard. The percentage of repetitions made by each subject was divided by the possible number of repetitions he or she could have made, to determine the level of plagiarism for the initial-generation phase. Although the level of repetitions (i.e., initial-generation plagiarism) on the initial-generation task was slightly higher for older adults (see Table 1), the age group difference was not significant $\left[F(1,59)=0.61, M S_{\mathrm{e}}=0.03\right]$, though these rates appear to be at floor.

Generate-new task. Generate-new plagiarism was calculated as the proportion of exemplars on the generatenew task that had been generated during the initialgeneration phase. These data are presented in Table 1. Of primary interest, results from the generate-new task indicate that older adults were more likely to plagiarize than were young adults $\left[F(1,59)=20.73, M S_{\mathrm{e}}=0.07\right]$. Self-plagiarisms across the task were nearly nonexistent, with only one self-plagiarism for each age group on the generate-new task; the greatest difference between age groups was the tendency for older adults to repeat other people's responses. We note that the generate-new plagiarism rate for young adults was low (3.9\%), but this is consistent with that found in previous research (Brown \& Murphy, 1989; Macrae et al., 1999), and is significantly greater than the $1.6 \%$ rate of recall repetitions used as a baseline for comparison in other studies (Brown \& Murphy, 1989) $[t(31)=3.14]$. Significantly more older adults (24 of $29 ; 83 \%$ ) plagiarized at least one exemplar than did young adults $(18$ of $32 ; 56 \%)\left[\chi^{2}(1, N=61)=4.61\right]$.

Recall task. Memory measures for Experiment 1 are presented in Table 1. In Experiment 1, each of six subjects generated two exemplars for each category, so recall is examined separately for self- and other-generated exemplars. Young adults recalled more words that they themselves generated, as well as more words that other subjects had generated. An ANOVA with age group (young, older) as a between-subjects variable was conducted for each performance measure and showed that young adults recalled

Table 1

Results of Recall and Generate-New Tasks for Experiment 1

\begin{tabular}{lccccc}
\hline & \multicolumn{4}{c}{ Age Group } \\
\cline { 2 - 3 } \multicolumn{1}{c}{ Response Type } & Young Adults & & \multicolumn{2}{c}{ Older Adults } \\
\cline { 2 - 3 } \cline { 5 - 6 } \multicolumn{1}{c}{} & $M$ & $S D$ & & $M$ & $S D$ \\
\hline Initial-generation plagiarism & .022 & .042 & & .031 & .054 \\
Recall-self & .979 & .078 & & .611 & .211 \\
Recall-other & .593 & .083 & & .450 & .091 \\
False recall & .016 & .021 & & .079 & .079 \\
Generate-new plagiarism & .039 & .044 & .109 & .074 \\
\hline
\end{tabular}

more self-generated exemplars $\left[F(1,59)=74.38, M S_{\mathrm{e}}=\right.$ 1.87], and more other-generated exemplars than did older adults $\left[F(1,59)=41.21, M S_{\mathrm{e}}=0.31\right]$.

False recall was defined as responses on the recall task that were not generated during initial generation, and was calculated by taking the number of these intrusions for a given subject and dividing it by the total number of responses for a given subject. False recall was more than four times greater for older adults than for young adults, who appeared to be at floor. This age group difference was also significant $\left[F(1,59)=19.10, M S_{\mathrm{e}}=0.06\right]$. We should note that in all experiments analyzing the raw number of intrusions led to the same statistical conclusions as analyzing the proportional measure.

Finally, young adults recalled a higher percentage of Stroop color words on the Stroop span task $(M=.77$, $S D=.10)$ than did older adults $(M=.50, S D=.19)$ $\left[F(1,59)=49.58, M S_{\mathrm{e}}=1.10\right]$.

Regression analysis. The regression analysis examining the relation between working memory capacity, episodic recall, and memory errors was delayed until after Experiment 2. The results from Experiments 1 and 2 were then combined and analyzed together, to increase power.

In sum, when asked to generate new exemplars, older adults were more likely to plagiarize responses that had previously been generated by other subjects. Older adults were also more likely to falsely recall exemplars that had not been generated earlier. The finding that generate-new plagiarism increased for older adults is consistent with the notion that avoiding generate-new plagiarism requires deliberate, systematic decision criteria. False recall was also more likely for older adults than for young adults, which is consistent with other recent reports in the literature (Balota et al., 1999; Butler et al., 2004).

\section{EXPERIMENT 2}

Experiment 1 established that older adults were more likely to accidentally plagiarize others' responses when generating new responses. We have suggested that this is the result of age-related changes in effortful, deliberate decision processes, but there are possible alternative explanations that have nothing to do with memory or decision processes. One possibility is that demand characteristics are driving age differences in inadvertent plagiarism; specifically, that older adults may have more difficulty generating four new exemplars on the generate-new task than do young adults, but, rather than failing to complete the task, they may simply be outputting exemplars that they knew to be old, to comply with task instructions. Others have suggested that this demand characteristic, whereby subjects deliberately repeat responses, may influence inadvertent plagiarism (Brédart et al., 2003; Tenpenny et al., 1998). To determine if this was the case, we asked subjects to rate their confidence for items output on the generatenew task. If demand characteristics influence age difference in generate-new plagiarism, older adults' confidence should be lower for plagiarized responses, because some subset of those responses were known to be errors. This is an especially important issue in this paradigm, because 
one of the more interesting aspects of inadvertent plagiarism is that people presumably believe that the information that they output is their own original product.

Additionally, we used the more typical inadvertent plagiarism procedure, whereby subjects were asked only to recall the exemplars that they themselves generated, and were required to complete the recall and generatenew tasks on the same answer sheet (see Brown \& Halliday, 1991). These design changes allowed us to assess recall-own plagiarism as well as generate-new plagiarism. Because recall-own plagiarism is a source memory error, we expected these errors to be greater for older adults, based on earlier findings of age-related declines in source memory (Glisky et al., 2001; McIntyre \& Craik, 1987).

\begin{abstract}
Method
Subjects. Subjects, recruited from the same subject pools as in Experiment 1, were 32 young (mean age $=19.1, S D=1.5$ ) and 32 older (mean age $=71.0, S D=5.5$ ) adults.

Materials and Procedure. Materials and procedure were the same as in Experiment 1, except where noted. Subjects in each age group were tested in groups of four. The recall-own and generatenew tasks were combined on a single sheet of paper (see Brown $\&$ Halliday, 1991). Thus, in Experiment 2, the tasks were given together. In the initial-generation phase, each subject alternated generating exemplars from each of six categories. Each subject was called upon four times per category, for a total of 16 exemplars per category. The categories used were sports, vegetables, musical instruments, fruit, clothing, and four-legged animals. Subjects were called upon to generate exemplars in a random order for half the groups, or in serial order (A, B, C, D). Results did not differ as a result of this manipulation, which will not be discussed further.

Approximately $30 \mathrm{~min}$ after initial generation, subjects in the recall group were asked to recall the exemplars that they themselves generated during the initial-generation phase (i.e., recall-own task), and to generate four new exemplars (the generate-new task). These tasks were given simultaneously, each category name being presented on a sheet with two columns below-one column in which subjects should recall the words they generated, the other in which they should write four new exemplars. Subjects were also asked to rate how confident they were that their generate-new exemplars were actually new. Confidence was rated on a three-point scale: $1=$ somewhat confident; $2=$ confident $; 3=$ very confident . Subjects were given $15 \mathrm{~min}$ for the combined recall-own-generate-new task. During the interval between initial generation and the recall-own task, subjects completed Shipley vocabulary, letter comparison, and Stroop span, all of which took about $30 \mathrm{~min}$. A demographic questionnaire was given after the generate-new task. No confederates were included in the initial-generation session.
\end{abstract}

\section{Results}

Two young adults and one older adult were excluded for failing to follow instructions or not generating four responses for each category.

Initial-generation phase. During the initial-generation phase, older adults made significantly more repetitions than did young adults $\left[F(1,59)=6.19, M S_{\mathrm{e}}=0.009\right]$. The unreliability of this effect across experiments is likely due to floor effects.

Recall-own task. The results for Experiment 2 are presented in Table 2. Again, young adults recalled more exemplars than older adults. This observation was confirmed by an ANOVA with age group (young, older) as a between-subjects variable $\left[F(1,59)=41.76, M S_{\mathrm{e}}=0.57\right]$.
Older adults also erroneously recalled exemplars that others had generated earlier more often than young adults (recall-own plagiarism) $\left[F(1,59)=10.92, M S_{\mathrm{e}}=0.05\right]$. On the few occasions when two subjects had generated the same item during initial generation, the item was considered self-generated for purposes of scoring recall and recall-own plagiarism. Finally, older adults erroneously recalled exemplars that had not been generated earlier (i.e., false recall) more often than young adults $[F(1,59)=$ $\left.8.95, M S_{\mathrm{e}}=0.04\right]$.

Generate-new task. Older adults were more likely to make repetitions when generating new responses than were young adults (generate-new plagiarism) $[F(1,59)=5.65$, $\left.M S_{\mathrm{e}}=0.03\right]$. Self-plagiarism across the task was significantly greater for older adults $\left[F(1,59)=11.95, M S_{\mathrm{e}}=\right.$ $0.0001]$, though again these errors were rare $(<2 \%$ for both age groups). Other-repetitions were also greater for older adults $\left[F(1,59)=6.21, M S_{\mathrm{e}}=0.03\right]$, consistent with the previous experiment. In contrast to the previous experiment, however, the proportion of subjects who inadvertently plagiarized a previous response was roughly equivalent for both age groups. There were no differences in the proportion of older adults ( 24 of $30 ; 80 \%$ ) and the proportion of young adults $(24$ of $31 ; 81 \%)$ who plagiarized at least one exemplar $\left(\chi^{2}=0.004\right)$. Thus, even when an equal number of subjects in each age group showed some level of inadvertent plagiarism, there was still an age-related increase in the overall level of inadvertent plagiarism.

Confidence. According to a demand-characteristic explanation of age differences in inadvertent plagiarism, older adults should be less confident in their plagiarized responses than young adults. (The confidence data are presented in Table 3.) In order to examine age differences in confidence, we investigated whether the distribution of confidence scores for correct items and plagiarized items differed as a function of age. An examination of Table 3 indicates that young adults were more confident in their correct (i.e., new) responses, giving more highest confidence responses and fewer lower confidence responses. The difference between age groups was significant (Kolmogorov-Smirnov $Z=1.65, p<.01$ ). By contrast, there were no age differences in confidence for plagiarized responses, with nearly identical percentages of each response type for both age groups (Kolmogorov-Smirnov $Z=0.008)$. Additionally, confidence was significantly higher for correct items than for incorrect items (Wilcoxon $Z=8.51, p<.01)$. We should also note that plagiarized items were at least as likely to be given a rating of 3 (i.e.,

Table 2

Results of Recall and Generate-New Tasks for Experiment 2

\begin{tabular}{lccccc}
\hline & \multicolumn{4}{c}{ Age Group } \\
\cline { 2 - 3 } \multicolumn{1}{c}{ Response Type } & \multicolumn{2}{c}{ Young Adults } & & Older Adults \\
\cline { 2 - 3 } \cline { 5 - 6 } \multicolumn{1}{c}{} & $M$ & $S D$ & & $S D$ \\
\hline Initial-generation plagiarism & .007 & .014 & & .018 & .027 \\
Recall own & .929 & .080 & & .737 & .145 \\
Recall-own plagiarism & .018 & .029 & & .078 & .096 \\
False recall & .019 & .033 & & .070 & .089 \\
Generate-new plagiarism & .063 & .055 & .104 & .079 \\
\hline
\end{tabular}


the highest confidence rating) as to be given a rating of either 1 or 2 for both young and older adults.

Output position for plagiarized responses on the generate-new task. One additional analysis that provides information about the demand characteristic explanation of age differences in inadvertent plagiarism is the output position of the plagiarized responses on the generate-new task. Plagiarized responses on the generate-new task can occur in position $1,2,3$, or 4 for each category. If subjects are simply outputting items they know to be plagiarized because they want to comply with task instructions and cannot think of any more new exemplars, one would expect these errors to occur in the latter output positions on the generate-new task. Thus, we examined, separately for young and older adults, whether plagiarized responses occurred in one of the first two output positions or last two output positions. To reiterate, according to the demand characteristic explanation of age differences in generatenew plagiarism, plagiarized responses should be more likely for older adults in positions 3 and 4 (i.e., on the latter half of the output positions). However, the data suggest that this was not the case. For young adults, $54 \%$ of their plagiarized responses occurred in the latter positions $\left(\chi^{2}=\right.$ $0.31)$, whereas for older adults, the corresponding value was $47 \%\left(\chi^{2}=0.32\right)$. An identical analysis performed on the data from Experiment 1 showed similar results. For young adults, again $54 \%$ of their plagiarized responses occurred in the latter positions $\left(\chi^{2}=0.15\right)$, whereas for older adults, the corresponding value was $40 \%\left(\chi^{2}=\right.$ $2.48)$. None of these values reached conventional significance levels, but if anything, the trend in the data indicated that older adults were less likely to plagiarize responses in the latter output positions. These data cast considerable doubt on the demand characteristic explanation of age differences in generate-new plagiarism.

Finally, young adults recalled a higher proportion of Stroop color words on the Stroop span task $(M=.74$, $S D=.13)$ than did older adults $(M=.48, S D=.14)$ $\left[F(1,59)=52.62, M S_{\mathrm{e}}=0.98\right]$.

\section{Discussion}

In Experiment 2, we replicated the age difference in inadvertent plagiarism on the generate-new task and also found that older adults were more likely to plagiarize others on the recall-own task. These findings suggest that systematic decision processes were required to avoid inadvertent plagiarism on both of these tasks, and also replicates previous research showing age-related decreases in source

Table 3

Percentage of Each Confidence Rating for New Items and Plagiarized Items on the Generate-New Task in Experiment 2

\begin{tabular}{lrcc}
\hline & \multicolumn{3}{c}{ Confidence Rating } \\
\cline { 2 - 4 } \multicolumn{1}{c}{ Response Type } & Low & Medium & High \\
\hline Young Adults & 3.8 & 10.1 & 86.1 \\
$\quad$ New words (correct) & 40.3 & 19.4 & 40.3 \\
$\quad$ Plagiarized words (errors) & & & \\
Older Adults & 10.2 & 11.7 & 78.1 \\
$\quad$ New words (correct) & 40.4 & 19.1 & 40.4 \\
$\quad$ Plagiarized words (errors) & &
\end{tabular}

recall (Schacter et al., 1991). Confidence was lower for plagiarized items than for correctly generated new items, consistent with previous findings (Brédart et al., 2003; Brown \& Murphy, 1989). Confidence for correct items was higher for young adults than for older adults, but there were no age differences in confidence ratings for items inadvertently plagiarized on the generate-new task. Thus, the age-related increase in plagiarism found here and in Experiment 1 was probably not due to demand characteristics.

\section{Regression Analysis for Experiments 1 and 2}

To examine the nature of the age differences found in generate-new plagiarism and false recall in Experiments 1 and 2, we used hierarchical regression to examine the proportion of variance in these errors that was accounted for by recall and Stroop span. After controlling for recall and Stroop span, we also examined whether age accounted for additional variance. If age did not account for significant variance beyond recall and working memory, this suggests that those processes mediate the age-related changes in the outcome measure. We used hierarchical regression because we wanted to examine the unique variance associated with each predictor, after controlling for the shared variance among them. For example, working memory capacity and recall were correlated $(r=.53)$. Furthermore, age was related to both recall and working memory capacity (both $r \mathrm{~s}=-.71$ ). Note also that each of these predictors alone showed significant correlations with generate-new plagiarism, with zero-order correlations $(r)$ of .41, -.52 , and -.42 for age, recall, and Stroop span, respectively. The same was true of false recall, with correlations of .47, -.51 , and -.49 for age, recall, and Stroop span, respectively. Thus, hierarchical regression allowed an examination of the relation between the variables after controlling for multicollinearity among the variables. (Note that we did not examine recall-own plagiarism in these analyses, because this measure was only available for Experiment 2, giving us little power to compute a reliable model.)

We began by considering the role of these measures in predicting generate-new plagiarism. For each subject, the percent recalled (recall) was converted to $z$ scores within each experiment, and the data from both experiments were combined. This allowed us to use more subjects in our regression model, increasing power and reliability. A total of 62 young adults and 60 older adults were included in the analysis $(N=122)$. To reiterate our hypotheses: It was possible that the age differences in generate-new plagiarism were simply due to the age-related deficit in recollecting studied items. While this is an intuitive prediction, it is also possible that working memory capacity accounts for unique variance above and beyond that of recall, due to the role of controlled attention in monitoring for errors (McCabe $\&$ Smith, 2002). The results of the analyses for generatenew plagiarism are shown in Table 4. In Model 1, recall was entered into the regression equation first, followed by Stroop span, and finally, age. The second column shows the amount of variance accounted for by the model at each step, whereas the third column shows the amount of additional variance that is accounted for by adding the variable listed in that row. Results indicate that recall accounted for 
a substantial proportion of variance $\left(R^{2}=.262\right)$, but that Stroop span still accounted for a significant proportion of additional variance after controlling for recall $\left(R^{2}=.033\right)$. Age was no longer related to generate-new plagiarism after recall and Stroop span were accounted for $\left(R^{2}=.002\right)$, suggesting that the age differences in generate-new plagiarism were completely explained by age differences in episodic recall and working memory capacity. Model 2 was identical, but Stroop span was entered first, followed by recall, in order to examine the amount of unique variance accounted for by recall. In this model, recall accounted for a significant proportion of additional variance after controlling for Stroop span $\left(R^{2}=.116\right)$, but again age was no longer related to generate-new plagiarism after Stroop span and recall were accounted for $\left(R^{2}=.002\right)$.

Table 5 shows the results of the regression analysis for false recall. Model 1 for false recall, with recall entered first, was very similar to the model for generate-new plagiarism. Recall accounted for a substantial amount of variance $\left(R^{2}=.259\right)$, but Stroop span accounted for a significant amount of additional variance $\left(R^{2}=.070\right)$, after recall was entered. Model 2 also led to similar results. After controlling for Stroop span, recall still accounted for a significant portion of unique variance $\left(R^{2}=.086\right)$. Finally, age no longer predicted false recall in either model, after recall and Stroop span were accounted for $\left(R^{2}=.000\right)$.

In summary, both recall and working memory capacity were involved in reducing memory errors. Combined, these measures completely eliminated the age difference in generate-new plagiarism and false recall. Thus, the monitoring involved in avoiding memory errors of both types was related to the ability to retrieve the exemplars that were actually studied, suggesting that recalling what was presented allowed subjects to avoid repeating what was presented, and also to reject what was not presented. However, there appear to be attentional demands involved in monitoring for memory errors beyond those involved in episodic recall, as indicated by the unique variance associated with working memory capacity after controlling for recall. It appears that working memory capacity is somewhat less important in accounting for generate-new plagiarism than recall was, as indicated by the amount of unique variance accounted for by recall and Stroop span (.116 and .033, respectively).

Table 4

Hierarchical Regression Model Predicting Generate-New Plagiarism in Experiments 1 and 2

\begin{tabular}{|c|c|c|c|}
\hline Step & Predictor & $R^{2}$ & $\Delta R^{2}$ \\
\hline \multicolumn{4}{|c|}{ Model 1: Recall First } \\
\hline 1 & Recall & $.262^{* *}$ & - \\
\hline 2 & Stroop span & $.295^{* *}$ & $.033^{*}$ \\
\hline 3 & Age & $.298^{* *}$ & .002 \\
\hline \multicolumn{4}{|c|}{ Model 2: Stroop Span First } \\
\hline 1 & Stroop span & $.180^{* *}$ & - \\
\hline 2 & Recall & $.295^{* *}$ & $.116^{*}$ \\
\hline 3 & Age & $.298^{* *}$ & .002 \\
\hline
\end{tabular}

Note- $R^{2}$ represents the proportion of variance accounted for by the predictors in the model. $\Delta R^{2}$ represents the increase in the proportion of variance accounted for by adding that predictor to the previous predictor(s). ${ }^{*} p<.05 .{ }^{* *} p<.01$.
Table 5

Hierarchical Regression Model Predicting False Recall in Experiments 1 and 2

\begin{tabular}{|c|c|c|c|}
\hline Step & Predictor & $R^{2}$ & $\Delta R^{2}$ \\
\hline \multicolumn{4}{|c|}{ Model 1: Recall First } \\
\hline 1 & Recall & $.259^{* *}$ & - \\
\hline 2 & Stroop span & $.329^{* *}$ & $.070^{* *}$ \\
\hline 3 & Age & $.329^{* *}$ & .000 \\
\hline \multicolumn{4}{|c|}{ Model 2: Stroop Span First } \\
\hline 1 & Stroop span & $.243^{* *}$ & - \\
\hline 2 & Recall & $.329^{* *}$ & $.086^{* *}$ \\
\hline 3 & Age & $.329^{* *}$ & .000 \\
\hline
\end{tabular}

Note- $R^{2}$ represents the proportion of variance accounted for by the predictors in the model. $\Delta R^{2}$ represents the increase in the proportion of variance accounted for by adding that predictor to the previous predictor(s). ${ }^{* *} p<.01$.

By contrast, similar amounts of unique variance were accounted for by recall and Stroop span in our models predicting false recall (.086 and .070, respectively).

\section{GENERAL DISCUSSION}

In two experiments, when asked to generate new items (i.e., generate-new plagiarism), older adults were more likely than young adults to inadvertently plagiarize exemplars they had encountered earlier. This age difference in generate-new plagiarism occurred when subjects were asked to generate new items before recalling the exemplars that had been generated, as in Experiment 1, or when recall and generate-new were done simultaneously, as in Experiment 2. Although confidence was greater for exemplars that were actually new, as compared with plagiarized responses, there were no age differences in the confidence of these responses in Experiment 2. Contrary to the predictions of a demand-characteristic account of the effect (e.g., Tenpenny et al., 1998), plagiarized responses were equally likely for both young and older adults to be output in the early and latter position for categories on the generate-new task. This suggests that subjects did not knowingly plagiarize responses on the generate-new task simply to comply with task instructions, and that demand characteristics cannot explain the age differences we report. Regression analyses revealed that the age differences found in generate-new plagiarism were eliminated when recall of studied exemplars and working memory capacity were controlled. Both of these variables accounted for unique variance in these memory errors, suggesting that avoiding generate-new plagiarism requires attentional control, and is also dependent on retrieval of previously presented exemplars.

Other memory errors were also more likely for older adults than for young adults. Inadvertent plagiarism on the recall-own task (i.e., recall-own plagiarism), in which subjects recall others' responses as their own, was more likely for older adults (Experiment 2), replicating previous reports of age-related increases in source-memory errors in recall (Brown et al., 1995; Schacter et al., 1991). False recall was also more likely for older adults in both experiments, replicating previous studies showing age- 
related increases in false recall (Balota et al., 1999; Butler et al., 2004; Norman \& Schacter, 1997). Consistent with the regression model for generate-new plagiarism, false recall was related to recall of studied exemplars and working memory capacity; controlling for these variables eliminated the age difference in false recall. Thus, the present results provide evidence that avoiding inadvertent plagiarism and avoiding false recall are reliant on similar retrieval processes and attentional resources.

\section{Age Differences in Inadvertent Plagiarism on the Generate-New Task}

The present results cast doubt on the notion that generate-new plagiarism simply involves failures to assess the familiarity of previously encountered items. Thus, despite the literature indicating that recall-own plagiarism and generate-new plagiarism rely on different types of information, the present results suggest similar cognitive demands for both tasks, at least in terms of the decision processes involved. The regression analyses further support this conclusion and suggest that systematic decision processes are involved in avoiding inadvertent plagiarism (Marsh et al., 1997).

Marsh et al. (1997) noted that generate-new plagiarism may require systematic decision processes because source is being monitored as a secondary task when subjects are attempting to generate new items. Typically, in everyday life, we are not vigilantly monitoring for errors such as inadvertent plagiarism; rather, as a goal in and of itself, we attempt to generate creative products; monitoring is secondary to this task (Jacoby, Kelley, \& Dywan, 1989). For example, Jacoby and colleagues have explained the false-fame paradigm as involving source monitoring as a secondary process (Dywan \& Jacoby, 1990; Jacoby et al., 1989). In the false-fame paradigm, subjects are first asked to pronounce nonfamous names and are later given a separate fame judgment task, on which they are asked to identify famous names. Findings indicate that subjects are more likely to judge a nonfamous name famous if they had pronounced it earlier, and furthermore, that older adults are more likely to make these errors. Generate-new plagiarism is similar, in that previous encounters with exemplars make the exemplars more familiar and increase the likelihood that they will be output as an error, but these errors differ from false fame in at least two important ways. First, subjects in the inadvertent-plagiarism paradigm believe that they themselves are the source of the exemplar being output, at least in the experimental context. Second, the age-related increases in false-fame errors have been attributed to age differences in decision criteria, because encouraging more stringent decision criteria eliminates the age difference in these errors (Multhaup, 1995). Given the strong nature of the instruction to exclude initially generated responses on the generate-new task, a decision criteria explanation of the present results seems unlikely.

Based on the experimental data we report, as well as the regression analyses, we believe the primary cause of the age differences in plagiarism is the nature of the retrieval processes involved in monitoring for plagiarism errors. Age differences in generate-new plagiarism were related to age-related changes in episodic memory as well as to a measure of working memory capacity. At the outset, we suggested that findings of age-related increases in generate-new plagiarism could be due to age-related declines in recall of studied exemplars, age-related declines in working memory capacity, or both. The regression analysis revealed that both of these factors were important to reducing generate-new plagiarism. Perhaps the finding that recall was related to generate-new plagiarism is somewhat obvious, with better recall of what was presented allowing one to reject these items on the generate-new task. However, the finding that working memory capacity was inversely related to generate-new plagiarism suggests that attentional resources beyond those involved in retrieval of studied exemplars were important to avoiding these memory errors. We believe that working memory capacity is important for reducing generate-new plagiarism, and memory errors more generally, because attentional resources are required for effective discrimination between similar sources of activation (Balota et al., 1999; McCabe \& Smith, 2002). The present results suggest that the generate-new task involves consciously retrieving exemplars from the study context and comparing them with exemplars currently activated during the generatenew task. This comparison process, whereby memory is an object of reflection, requires attentional resources (Jacoby et al., 1989; Johnson et al., 1993; Moscovitch \& Winocur, 1992).

Jacoby and colleagues have recently proposed that some age-related increases in memory errors may result from older adults being "captured" by information that is highly accessible (Jacoby, Bishara, Hessels, \& Toth, 2005). They found that older adults were much more likely than young adults to make memory errors when they generated familiar targets in response to experimenter-provided cues, despite those targets being incorrect in that particular experimental context. They argued that the act of retrieval itself precluded the opportunity to recollect information from the study episode, resulting in increased memory errors for older adults. The act of retrieval may be a source of interference, acting like misinformation introduced between study and test, which can cause difficulty in reality monitoring for older adults (e.g., "Is this item familiar because I heard it earlier, or because I just thought of it now?"). Furthermore, Jacoby et al. found that performance on the Wisconsin Card Sorting task, a popular measure of executive functioning (Rhodes, 2004), was related to these errors. Although speculative, the "capture" explanation proposed by Jacoby et al. suggests a possible mechanism for the age-related increases in generate-new plagiarism found in the present study. Older adults may be repeatedly generating the same exemplars when considering items to output, and these repeated acts of generation may act as a source of interference, making it more difficult to recollect whether a familiar item was actually studied earlier. Furthermore, the finding that an executive function measure predicted these types of errors in the Jacoby et al. experiments is consistent with the working memory capacity data in the present study, at least to the extent that executive functioning and working memory capacity 
are similar constructs (McCabe et al., 2005). More work will be needed to examine whether this retrieval capture mechanism influences age-related increases in memory errors like those seen in the present study.

\section{Age Differences in False Recall}

In addition to examining age differences in inadvertent plagiarism, we examined the false recall of category exemplars on the recall tasks. The results were clear across both experiments: Older adults were more likely to falsely recall exemplars that had not been presented. These results are consistent with other recent reports in the literature (Meade \& Roediger, 2006), and are also consistent with the results from research using the DRM false-memory paradigm (Balota et al., 1999; Butler et al., 2004; Norman \& Schacter, 1997), in which associates of studied items are falsely recalled as having been presented. The activation monitoring account of false memories provides a straightforward explanation of these results (McDermott \& Watson, 2001; Roediger et al., 2001). According to this explanation, during the initial generation of exemplars, associates of category exemplars are likely to come to mind due to spreading activation in semantic networks. If these associates fit the category, but are not output during the initial generation phase, they may later be falsely recalled if reality monitoring processes are not effectively engaged (Meade \& Roediger, 2006). This account of false memories suggests that repeated activation of related words will result in false recall if the associative activation is strong enough and recall of actual events, which is required for effective source monitoring, is low (McDermott \& Watson, 2001; Roediger et al., 2001). The finding that recall of studied exemplars was inversely related to false recall is consistent with this explanation. The finding that working memory capacity predicted false recall of category exemplars is also consistent with the notion that monitoring processes are crucial to editing out false memories, and is also consistent with previous research on false memories (e.g., McCabe \& Smith, 2002). Of course, in the present study we do not know the exact mechanism that is driving the correlation between Stroop span and memory errors, only that there is some overlap in the nature of the processing between the two tasks. Thus, it could be that the inhibitory demands involved in the tasks are similar, or that both tasks require the suppression of proactive interference, or that both tasks require subjects to maintain task goals in the face of distraction. In any event, all of these possible reasons for the correlation suggest that the ability to control attention is important for avoiding memory errors.

\section{Plagiarism Outside the Laboratory}

Although the category-generation paradigm used here is obviously not a direct analogy to plagiarism outside the laboratory, we do suggest that it is ecologically valid; that is, the inadvertent-plagiarism paradigm is believed to capture the memory processes involved in inadvertent plagiarism in the real world (though other processes are certainly involved, such as expertise in a domain, social/ professional costs associated with plagiarism, etc.). Thus, the present data are relevant to situations in which blame is placed on plagiarists in the areas of music, film, literature, academics, and art. Perhaps because the primary safeguard against plagiarism in these fields is the integrity of those who would offend, there are few more grave transgressions than "stealing" the work of another. Typically, when plagiarism is discovered, the assumption is that the intent was malicious, with little regard for the role of unconscious processes in creating these problems (Mallon, 1989). In fact, unconscious influences are often given as the reason for plagiarism, either explicitly or implicitly. For example, Mark Twain, who admitted to having inadvertently plagiarized nearly an entire book dedication from Oliver Wendell Holmes, claimed that "substantially all ideas are secondhand, consciously and unconsciously drawn from a million outside sources, and daily used by the garnerer with a pride and satisfaction born of the superstition that he originated them" (Braddy, 1934, p. 162). Perhaps we should be impressed that our cognitive system typically allows us to be so effective at avoiding plagiarism, rather than reacting with indignation at the mere possibility that the creative product of one person was duplicated by another.

\section{AUTHOR NOTE}

Please address correspondence to D. P. McCabe, Department of Psychology, Colorado State University, Campus Box 1876, Fort Collins, CO 80523-1876 (e-mail: david.mccabe@colostate.edu).

\section{REFERENCES}

Balota, D. A., Cortese, M. J., Duchek, J. M., Adams, D., Roediger, H. L., III, McDermott, K. B., \& Yerys, B. E. (1999). Veridical and false memories in healthy older adults and in dementia of the Alzheimer's type. Cognitive Neuropsychology, 16, 361-384.

Balota, D. A., Dolan, P. O., \& DucheK, J. M. (2000). Memory changes in healthy older adults. In E. Tulving \& F. I. M. Craik (Eds.), Oxford handbook of memory (pp. 395-410). Oxford: Oxford University Press.

Battig, W. F., \& Montague, W. E. (1969). Category norms for verbal items in 56 categories: A replication and extension of the Connecticut category norms. Journal of Experimental Psychology, 80(3, Pt. 2).

Benjamin, A. S. (2001). On the dual effects of repetition on false recognition. Journal of Experimental Psychology: Learning, Memory, \& Cognition, 27, 941-947.

Braddy, N. (1934). Anne Sullivan Macy: The story behind Helen Keller. Garden City, NY: Doubleday, Doran.

Brainerd, C. J., Reyna, V. F., \& KneER, R. (1995). False-recognition reversal: When similarity is distinctive. Journal of Memory \& Language, 34, 157-185.

Brédart, S., Lampinen, J. M., \& Defeldre, A.-C. (2003). Phenomenal characteristics of cryptomnesia. Memory, 11, 1-11.

Brown, A. S., \& Halliday, H. E. (1991). Cryptomnesia and source memory difficulties. American Journal of Psychology, 104, 475-490.

Brown, A. S., Jones, E. M., \& Davis, T. L. (1995). Age differences in conversational source monitoring. Psychology \& Aging, 10, 111-122.

Brown, A. S., \& Murphy, D. R. (1989). Cryptomnesia: Delineating inadvertent plagiarism. Journal of Experimental Psychology: Learning, Memory, \& Cognition, 15, 432-442.

Butler, K. M., McDaniel, M. A., Dornburg, C. C., Price, A. L., \& RoEdiger, H. L., III (2004). Age differences in veridical and false recall are not inevitable: The role of frontal lobe function. Psychonomic Bulletin \& Review, 11, 921-925.

Castel, A. D., \& Craik, F. I. M. (2003). The effects of aging and divided attention on memory for item and associative information. Psychology \& Aging, 18, 873-885.

Craik, F. I. M., \& Jennings, J. M. (1992). Human memory. In F. I. M. Craik \& T. A. Salthouse (Eds.), The handbook of aging and cognition (pp. 51-110). Hillsdale, NJ: Erlbaum. 
DEESE, J. (1959). On the prediction of occurrence of particular verbal intrusions in immediate recall. Journal of Experimental Psychology, $\mathbf{5 8}, 17-22$.

DYWAN, J., \& JACOBY, L. L. (1990). Effects of aging on source monitoring: Differences in susceptibility to false fame. Psychology \& Aging, 5, 379-387.

Engle, R. W., Tuholski, S. W., Laughlin, J. E., \& Conway, A. R. A (1999). Working memory, short-term memory and general fluid intelligence: A latent-variable approach. Journal of Experimental Psychology: General, 128, 309-331.

Glisky, E. L., Rubin, S. R., \& Davidson, P. S. R. (2001). Source memory in older adults: An encoding or retrieval problem? Journal of Experimental Psychology: Learning, Memory, \& Cognition, 27, 1131-1146.

Henkel, L. A., Johnson, M. K., \& De Leonardis, D. M. (1998). Aging and source monitoring: Cognitive processes and neuropsychological correlates. Journal of Experimental Psychology: General, 127, 251-268.

JACOBY, L. L. (1999). Ironic effects of repetition: Measuring age-related differences in memory. Journal of Experimental Psychology: Learning, Memory, \& Cognition, 25, 3-22.

Jacoby, L. L., Bishara, A. J., Hessels, S., \& Toth, J. P. (2005). Aging, subjective experience, and cognitive control: Dramatic false remembering by older adults. Journal of Experimental Psychology: General, 134, 131-148.

Jacoby, L. L., Kelley, C., \& Dywan, J. (1989). Memory attributions. In H. L. Roediger III \& F. I. M. Craik (Eds.), Varieties of memory and consciousness: Essays in honour of Endel Tulving (pp. 391-422). Hillsdale, NJ: Erlbaum.

JENNINGS, J. M., \& JACOBY, L. L. (1993). Automatic versus intentional uses of memory: Aging, attention, and control. Psychology \& Aging, 8, 283-293.

Johnson, M. K., Hashtroudi, S., \& Lindsay, D. S. (1993). Source monitoring. Psychological Bulletin, 114, 3-28.

LANDAU, J. D., \& Marsh, R. L. (1997). Monitoring source in an unconscious plagiarism paradigm. Psychonomic Bulletin \& Review, $\mathbf{4}$ 265-270.

Light, L. L., Patterson, M. M., Chung, C., \& Healy, M. R. (2004). Effects of repetition and response deadline on associative recognition in young and older adults. Memory \& Cognition, 32, 1182-1193.

LÖVDÉN, M. (2003). The episodic memory and inhibition accounts of age-related increases in false memories: A consistency check. Journal of Memory \& Language, 49, 268-283.

Macrae, C. N., Bodenhausen, G. V., \& Calvini, G. (1999). Contexts of cryptomnesia: May the source be with you. Social Cognition, 17, 273-297.

Mallon, T. (1989). Stolen words: Forays into the origins and ravages of plagiarism. New York: Ticknor \& Fields.

MARSH, R. L., \& Bower, G. H. (1993). Eliciting cryptomnesia: Unconscious plagiarism in a puzzle task. Journal of Experimental Psychology: Learning, Memory, \& Cognition, 19, 673-688.

Marsh, R. L., \& LANDAU, J. D. (1995). Item availability in cryptomnesia: Assessing its role in two paradigms of unconscious plagiarism. Journal of Experimental Psychology: Learning, Memory, \& Cognition, 21, 1568-1582.

Marsh, R. L., Landau, J. D., \& Hicks, J. L. (1997). Contributions of inadequate source monitoring to unconscious plagiarism during idea generation. Journal of Experimental Psychology: Learning, Memory, \& Cognition, 23, 886-897.

McCabe, D. P., Robertson, C. L., \& Smith, A. D. (2005). Age differences in Stroop interference in working memory. Journal of Clinical \& Experimental Neuropsychology, 27, 633-644.

McCabe, D. P., \& Smith, A. D. (2002). The effect of warnings on false memories in young and older adults. Memory \& Cognition, 30, 1065-1077.

McDermott, K. B., \& Watson, J. M. (2001). The rise and fall of false recall: The impact of presentation duration. Journal of Memory \& Language, 45, 160-176.

MCInTYRE, J. S., \& Craik, F. I. M. (1987). Age differences in memory for item and source information. Canadian Journal of Psychology, 41, 175-192.
Meade, M. L., \& Roediger, H. L., III (2006). The effect of forced recall on illusory recollection in younger and older adults. American Journal of Psychology, 119, 433-462.

Melton, A. W. (1967). Individual differences and theoretical process variables: General comments on the conference. In R. M. Gagné (Ed.), Learning and Individual Differences: A symposium of the Learning Research and Development Center. Columbus, $\mathrm{OH}$ : Merrill.

Moscovitch, M., \& Winocur, G. (1992). The neuropsychology of memory and aging. In F. I. M. Craik \& T. A. Salthouse (Eds.), The handbook of aging and cognition (pp. 315-372). Hillsdale, NJ: Erlbaum

Multhaup, K. S. (1995). Aging, source, and decision criteria: When false fame errors do and do not occur. Psychology \& Aging, 10, 492-497.

Naveh-Benjamin, M., \& Craik, F. I. M. (1995). Memory for context and its use in item memory: Comparisons of younger and older persons. Psychology \& Aging, 10, 284-293.

Norman, K. A., \& Schacter, D. L. (1997). False recognition in young and older adults: Exploring the characteristics of illusory memories. Memory \& Cognition, 25, 838-848.

Rhodes, M. G. (2004). Age-related differences in performance on the Wisconsin Card Sorting Test: A meta-analytic review. Psychology \& Aging, 19, 482-494.

Rhodes, M. G., \& Kelley, C. M. (2005). Executive processes, memory accuracy, and memory monitoring: An aging and individual difference analysis. Journal of Memory \& Language, 52, 578-594.

Roediger, H. L., III, \& McDermott, K. B. (1995). Creating false memories: Remembering words not presented in lists. Journal of Experimental Psychology: Learning, Memory, \& Cognition, 21, 803-814.

Roediger, H. L., III, Watson, J. M., McDermott, K. B., \& Gallo, D. A. (2001). Factors that determine false recall: A multiple regression analysis. Psychonomic Bulletin \& Review, 8, 385-407.

Salthouse, T. A., \& BabCocK, R. L. (1991). Decomposing adult age differences in working memory. Developmental Psychology, 27, 763-776.

Schacter, D. L., Israel, L., \& Racine, C. (1999). Suppressing false recognition in younger and older adults: The distinctiveness heuristic. Journal of Memory \& Language, 40, 1-24.

Schacter, D. L., Kaszniak, A. W., Kihlstrom, J. F., \& Valdiserri, M. (1991). The relation between source memory and aging. Psychology \& Aging, 6, 559-568.

SKINNER, B. F. (1983). Intellectual self-management in old age. American Psychologist, 38, 239-244.

Smith, A. D., \& EArLes, J. L. (1996). Memory changes in normal aging. In F. Blanchard-Fields \& T. Hess (Eds.), Perspectives on cognitive change in adulthood and aging (pp. 192-220). New York: McGraw-Hill.

Smith, S. M., Gerkens, D. R., Pierce, B. H., \& Choi, H. (2002). The roles of associative responses at study and semantically guided recollection at test in false memory: The Kirkpatrick and Deese hypotheses. Journal of Memory \& Language, 47, 436-447.

Sommers, M. S., \& HufF, L. M. (2003). The effects of age and dementia of the Alzheimer's type on phonological false memories. Psychology \& Aging, 18, 791-806.

Tenpenny, P. L., Keriazakos, M. S., Lew, G. S., \& Phelan, T. P. (1998). In search of inadvertent plagiarism. American Journal of Psychology, 111, 529-559.

UNDERWOOD, B. J. (1975). Individual differences as a crucible in theory construction. American Psychologist, 30, 128-134.

Watson, J. M., Bunting, M. F., Poole, B. J., \& Conway, A. R. A. (2005). Individual differences in susceptibility to false memory in the Deese-Roediger-McDermott paradigm. Journal of Experimental Psychology: Learning, Memory, \& Cognition, 31, 76-85.

Yonelinas, A. P. (2002). The nature of recollection and familiarity: A review of 30 years of research. Journal of Memory \& Language, 46, 441-517.

ZACHARY, R. A. (1986). Shipley Institute of Living Scale: Revised manual. Los Angeles: Western Psychological Services.

(Manuscript received August 14, 2005; revision accepted for publication November 30, 2005.) 